\title{
Research on Performance Test of Metering Chip
}

\author{
H. L. SUN \& K. J. ZHOU \\ Electric power research institute of Chongqing electric power grid, Chongqing, China
}

H. F. LUO

School of Electrical Engineering, Chongqing University, China

\begin{abstract}
For testing metering chip's function and performance, this paper has build a testing platform and done a lot of research, including testing dynamic range methods, reliability and security of metering chip. The error caused by other components is eliminated in this paper in this platform, comparing with traditional meter testing method. The testing platform can load various packages chips from various brands, and be able to not only evaluate performance of metering chips from different manufacturers, but also apply to function and performance test on bulk chips. In additional, a testing result of single phase metering chip V9281 is provided.
\end{abstract}

KEYWORD: Metering chip; Testing platform; Dynamic range; Reliability; Security

\section{BACKGROUND}

In order to ensure that intelligent meter is reliable in quality and accurate in metering, state grid established technical manual of intelligent meter in 2009 , so that it is possible to grade intelligent meters according to their performance test results and distinguish them. China association for intelligent meter industry and other research institutes have done intensive study on reasons could cause metering errors of intelligent meter. Except for the metering chip, other factors such as power supply and PCB design, will affect the measurement of performance. However, the performance testing system of the core component of intelligent meter -metering chip - has not been researched yet. State grid convened a workshop on identification of intelligent meter's core component in October 2013, the discussion[1-3]was focused on function, performance standard and grade standard of metering chip, but the problem that how to build a unified and fair testing platform still troubled representatives.

This paper aims at the performance test of metering chip, proposed the testing method for dynamic range, reliability and security of metering chip, provided a testing platform which is suit to test metering chips of various power supply and various packages.

\section{PERFORMANCE EVALUATION SYSTEM OF METERING CHIP}

The grade standard of intelligent meter established by state grid contains [4-5] fundamental error, timing error per day, power loss, voltage influence, influence of five times harmonic, temperature rise influence, self-heat influence, external magnet induction, error consistency, error change test, current variation, error repeatability, short time overcurrent influence, current loop impedance and so on. Power operations attributed the reasons of intelligent meter's invalidation to metering error, timing error per day, constant value of intelligent meter, communication function, liquid crystal display[6-7]. During the process of invitation for bids and operation, performance targets that state grid concerned are all directly related to the core component-metering chip, but since then there is no reference standard provided to evaluate the performance of a chip.

This paper takes calculating reliability and accuracy as fundamental principle and extends the range of metering chip according to its characters, divides testing into several parts listed as below:

1) Basic metering performance test. In operation of intelligent meter, electric quantity is the basis of settlement between power grid and customers, and the accuracy of electric quantity metering is directly related to metering performance. Metering accuracy of intelligent meter in wide range and start/shunt running performance has been the most concerned 
performance. To metering chips, that is to say, the linearity and stability of calculation error from the maximum input value reduces to the minimum value of metering chip.

2) Reliability and security test of metering chip. In the view of many design and operation department, the reliability and security of intelligent meter are even more important than metering accuracy. International institution such as IEC, VDE and NCB established a series of standards on the reliability and security of intelligent meter. IEC 60335, for example, it divides the reliability and security into 3 classes: Class A, the reliability and security is completely independent from software; Class B, the software is able to prevent unreliable or unsafe operations; Class $\mathrm{C}$, the software was specially designed to prevent harmful operation. Metering chips is contained in Class B. As to Class $\mathrm{B}$, it is necessary to find cooperation between hardware and software to improve reliability and security of system. It is required to do visiting privilege protection when software test and operate on key hardware devices of the micro control unit(hereinafter referred to as MCU) such as key register, stack pointer register, program pointer register, addressing and data paths, system clock, Non-volatile register and volatile register. Hardware devices themselves need to supply function of validation, warning and system reset to ensure the normal operation of software. As to metering chip, it should meet criterions of reliability test on integrated circuit and test the metering and communication performance of metering chip under boundary conditions of abnormal voltage supply, abnormal external devices, underload/overload, low power factor and harmonic waves according to the specific operating situation.

\section{TESTING PLATFORM OF METERING CHIP}

Except for metering chip, factors of power supply, sensors and PCB design may influence the metering performance of intelligent meter as well. As a result, it is necessary to design a platform which is able to solely load metering chips of various supply voltages in various packages so that we can evaluate the performance of metering chips without influenced by other factors.

It is available to complete test on metering chips in different packages with testing circuit board based on design of MCU and kinds of electric components. The tested metering chip is connected to testing circuit through sockets and loading circuit, after that, it will be tested by MCU programmed previously.

Testing circuit board of single phase metering chips joint developed by Chongqing electric power research institute is as shown in Fig.1. It contains fundamental function test (testing result output on liquid crystal display), signal-to-noise test of analog-digital converter (hereinafter referred to as ADC) and test of reliability and security under boundary and limiting conditions. Mainly function design is listed as below:

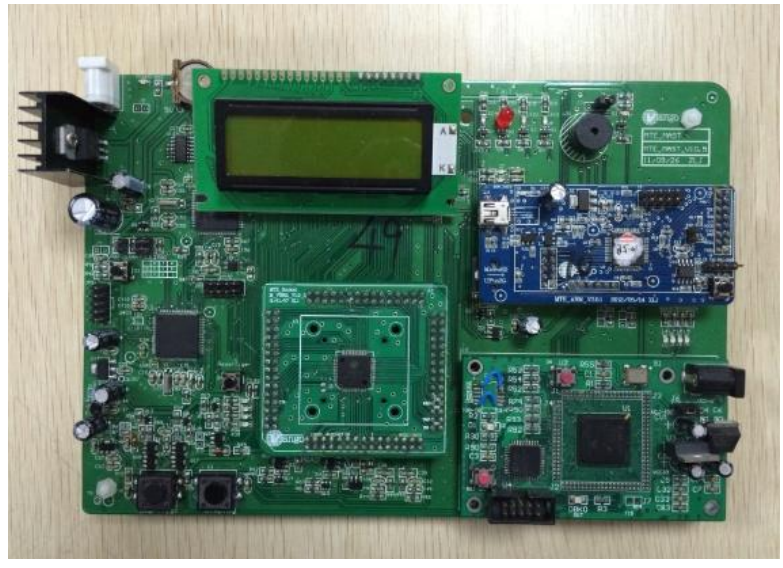

Fig.1 Testing circuit board of metering chips

1) In order to test metering chips of various power supplies, a steady DC voltage source which is continuously adjustable from $1.2 \mathrm{~V}$ to $6 \mathrm{~V}$ is needed.

2) Aiming at sample input of metering chip of intelligent meter, a steady AC signal source suit to testing board is necessary to generate a 3 phases AC signal whose amplitude and phase is continuously adjustable.

3) Aiming at the output of metering chip of intelligent meter, we need to supply a precision frequency meter to measure the interval between pulse CF outputs of metering chip besides visiting metering data through interface communication, and then calculate the accuracy of metering chip.

4) Aiming at different interface communication patterns of various metering chips applied in intelligent meters (usually universal asynchronous receiver transmitter (hereinafter referred to as UART) or serial peripheral interface(hereinafter referred to as SPI)). MCU is available to control by $\mathrm{C}$ program and can output signal of various transmission rate, various communication pattern and signal with communication interferences(such as rag, irregular duty ratio and so on) to communicate with metering chip according to communication protocol of metering chips.

5) In additional, other devices is needed to change the environmental temperature of metering chips so that the influence quantity test of metering chip applied in intelligent meter would be done successfully.

\subsection{Fundamental performance test of metering chip}

Fundamental performance of metering chip contains linearity error and stability error in rated dynamic range. In this test, we keep the input power frequency voltage of voltage input channel at a best 
value recommended by a chip data manual according to the deference among input voltage signals from various metering chips:

1) At the environmental temperature of $23^{\circ} \mathrm{C}$, set the angle between voltage input and current input be 0 degree and start the test from the rated maximum value of current input amplitude. Reduce the amplitude by a half and record the time interval of CF output with precision frequency meter for 5 times, take the average as the measuring value of linearity this testing point and the absolute value of the difference between maximum value and minimum value as the measuring value of stability, thus we can get the linearity curve and stability curve of the metering chip in its rated dynamical range (for example, 1:10000 or 1:5000).

2) Test on frequency influence quantity, harmonic wave influence quantity and temperature influence quantity of metering chip.

3) Power consumption test on metering chip.

The test result of V9281 is listed in Table 1:

Table 1 Fundamental performance test on metering chip V9281

\begin{tabular}{|l|l|l|}
\hline Testing project & Testing method & Testing performance \\
\hline \multirow{5}{*}{$\begin{array}{l}\text { Metering accuracy } \\
\text { test }\end{array}$} & \begin{tabular}{l} 
Testing point: refer to 3.1 \\
Frequency: $45 \mathrm{~Hz}, 49 \mathrm{~Hz}, 51 \mathrm{~Hz}$ and $55 \mathrm{~Hz}$. Testing \\
\cline { 2 - 3 }
\end{tabular} & $\begin{array}{l}\text { Linearity error less than } \pm 0.1 \% \text { in dynamical range } \\
\text { of } 5000: 1 .\end{array}$ \\
\cline { 2 - 3 } & $\begin{array}{l}\text { Error variation less than } 0.06 \% \text { compared to error } \\
\text { data of test of the second time. }\end{array}$ \\
\cline { 2 - 3 } & $\begin{array}{l}\text { Testing point: } 0.5 \mathrm{Imax} . \\
\text { Testing point: } 1.0,0.5 \mathrm{~L}, \mathrm{Ib} ; 1.0,5 \% \mathrm{Ib} .\end{array}$ & $\begin{array}{l}\text { Error variation less than } 0.05 \% \text { compared to error } \\
\text { data of test of the second time. }\end{array}$ \\
\hline Power consumption & Test on series of resistance and source. & $\begin{array}{l}\text { Temperature coefficient: } \leqq 0.01 \% / \mathrm{K}(\mathrm{error} \\
\text { variation } \leqq 0.6 \% \text { when temperature is high or low) }\end{array}$ \\
\hline
\end{tabular}

\subsection{Reliability test of metering chip}

Universal long-term reliability test on integrated circuit could refer to related testing standards of JEDEC. Reliability test in this paper is done to test the function and performance of metering chip under boundary conditions.

According to various input voltage signals of different metering chips, set effective values of $50 \mathrm{~Hz}$ input voltage of voltage input channel and current be the best value recommended by chip data manual, and the reliability test contains:

1) Influence test of source voltage. The test aims at energy supply variation of metering chip caused by voltage fluctuation and other unforeseen circumstances possible in operation of intelligent meter. In actual test, we respectively record the result on testing platform to figure out whether metering chip could normally meter and output CF be abnormal when source voltage is $\pm 10 \%, \pm 20 \%$, VDD and VSS.

Taking V9281 as testing chip, test 10 samples and testing data is shown in Table 2, the metering chip worked normally but the measurement accuracy declined.

2) Impact load test. Boundary conditions of this test are short-term overvoltage and overcurrent, it mainly reflects metering performance when motor starts or the supply voltage of grid fluctuate unusually. In this test, we supply $120 \%$ maximum input voltage and $120 \%$ maximum input current and observe CF output.
Table 2 Error test on source voltage fluctuation

\begin{tabular}{|c|c|c|c|c|c|}
\hline & \multicolumn{5}{|c|}{ Relative value of CF frequency error } \\
\hline Number & $2.64 \mathrm{~V}$ & $2.97 \mathrm{~V}$ & $3.3 \mathrm{~V}$ & $3.63 \mathrm{~V}$ & $3.96 \mathrm{~V}$ \\
\hline 1 & 0.217584 & 0.104999 & 0 & -0.01757 & -0.07386 \\
\hline 2 & 0.183741 & 0.093672 & 0 & -0.1297 & -0.15052 \\
\hline 3 & 0.305698 & 0.115734 & 0 & -0.05108 & -0.09778 \\
\hline 4 & 0.263124 & 0.123776 & 0 & -0.04911 & -0.06708 \\
\hline 5 & 0.167476 & 0.045275 & 0 & -0.09055 & -0.11619 \\
\hline 6 & 0.218352 & 0.119498 & 0 & 0.007146 & -0.04605 \\
\hline 7 & 0.229818 & 0.123626 & 0 & -0.0103 & -0.0523 \\
\hline 8 & 0.164098 & 0.037313 & 0 & -0.05938 & -0.08345 \\
\hline 9 & 0.186268 & 0.10189 & 0 & -0.01473 & -0.07363 \\
\hline 10 & 0.19338 & 0.099282 & 0 & 0.012759 & -0.04187 \\
\hline
\end{tabular}

Notes: Relative error is acquired with $\mathrm{CF}$ frequency under normal source voltage of V9281 as benchmark.

Taking V9281 as testing chip, test 10 samples and unusual $\mathrm{CF}$ frequency output is not found.

3) Short circuit test of crystal oscillator. The test mainly aims to the situation that external crystal oscillator stops or short circuits because of environment factors. Metering chip should avoid non-metering or abnormal metering situation. In this test, short circuit the external crystal oscillator and observe if the CF output is normal. 
Table 3 Short circuit test of crystal oscillator

\begin{tabular}{|c|c|c|c|}
\hline & \multicolumn{2}{|c|}{ CF frequency $(\mathrm{Hz})$} & \\
\hline Number & normal & Short circuit & restorability \\
\hline 1 & 256.754 & 278.867 & Yes \\
\hline 2 & 256.584 & 271.661 & Yes \\
\hline 3 & 257.858 & 270.926 & Yes \\
\hline 4 & 257.376 & 272.678 & Yes \\
\hline 5 & 255.613 & 269.732 & Yes \\
\hline 6 & 258.518 & 263.008 & Yes \\
\hline 7 & 258.727 & 269.528 & Yes \\
\hline 8 & 255.413 & 273.121 & Yes \\
\hline 9 & 257.435 & 273.579 & Yes \\
\hline 10 & 257.094 & 274.965 & Yes \\
\hline
\end{tabular}

To face the situation when external crystal oscillator works abnormal, V9281 is designed with internal monitoring interrupt tag and $\mathrm{RC}$ automatic switchover function, so it can meter normally after $\mathrm{CF}$ short circuited, but as a result, the accuracy of metering may fall.

We need to take the method of combination of hardware of software to face this situation occurs in actual operation of intelligent meter. On the one hand, testing and warning mechanism is needed in metering chip; on the other hand, in the system design MCU need to regularly check and response testing and warning mechanism to ensure the accuracy of metering chip.

4) Short circuit and open circuit test on external capacitor. The test simulates the situation that the external capacity of internal fundamental voltage source breakdown caused of oversize electrical stress in operation of intelligent meter. In the test, we respectively do short circuit test and open circuit and observe CF output.

Table 4 Short circuit and open circuit test on external capacitor

\begin{tabular}{|c|c|c|c|c|c|}
\hline & \multicolumn{5}{|c|}{ CF frequency } \\
\hline Number & REF level(V) & Normal & Short circuit & Open circuit & restorability \\
\hline 1 & 1.191 & 256.754 & $1.3 \mathrm{k}$ & 247.476 & Yes \\
\hline 2 & 1.1908 & 256.584 & $1.27 \mathrm{k}$ & 247.536 & Yes \\
\hline 3 & 1.1921 & 257.858 & $1.3 \mathrm{k}$ & 249.379 & Yes \\
\hline 4 & 1.1905 & 257.376 & $1.5 \mathrm{k}$ & 249.574 & Yes \\
\hline 5 & 1.1916 & 255.613 & $1.61 \mathrm{k}$ & 247.955 & Yes \\
\hline 6 & 1.1882 & 258.518 & $2.27 \mathrm{k}$ & 250.661 & Yes \\
\hline 7 & 1.189 & 258.727 & $2.286 \mathrm{k}$ & 250.731 & Yes \\
\hline 8 & 1.1927 & 255.413 & $1.31 \mathrm{k}$ & 247.561 & Yes \\
\hline 9 & 1.1915 & 257.435 & $2.4 \mathrm{k}$ & 249.927 & Yes \\
\hline 10 & 1.1924 & 257.094 & $1.79 \mathrm{k}$ & 249.605 & \\
\hline
\end{tabular}

It can be seen from the testing result that CF impulse frequency output would be obvious abnormal when the external fundamental voltage source short circuited. This situation can be properly dealt with MCU of upper computer or the mechanism of the metering chip. However, at early time after the short circuit occurs it is difficult to find since metering accuracy is still in normal range although it is getting worse. As a result, it is demanded to design metering chip in view of combination of hardware and software, that is to say metering chip should generate an interruption signal to do convenience for judge and deal of MCU when external capacitor works abnormally.

5) Test on communication reliability. This test reflects the communication reliability of metering chip under complex electromagnetic interferences. Based on the testing board of metering chip designed before, write program on upper PC computer in $\mathrm{C}$ language or assembly language and do kinds of programming operations to MCU controller of target testing board with computer interface presupposed and corresponding downloading tools, thus various communication test is available. Communication test inspects stability and communicating success rate of metering data when communicated in various transmission rates, different communication modes and with communication crosstalk added.

Since the chip V9281 tested applies UART interface, this test do test on common baud rates $1200 \mathrm{bps}, 2400 \mathrm{bps}$, 4800bps and $9600 \mathrm{bps}$ to evaluate the chip's suitability to non-synchronous of communication clock, the result is as shown in table5. 
Table 5 Test on UART communication baud rate range of V9281

\begin{tabular}{|c|c|c|c|c|}
\hline Rated baud rate & \multicolumn{2}{|c|}{ Minimum communication baud rate } & \multicolumn{2}{|c|}{ Maximum communication baud rate } \\
\hline 1200 & 1127 & $-6.08 \%$ & 1247 & $3.92 \%$ \\
\hline 2400 & 2253 & $-6.13 \%$ & 2497 & $4.04 \%$ \\
\hline 4800 & 4509 & $-6.06 \%$ & 4988 & $3.92 \%$ \\
\hline 9600 & 9028 & $-5.96 \%$ & 9955 & $3.70 \%$ \\
\hline
\end{tabular}

As we can infer from the test result on UART communication of V9281, the anti-noiseinterference ability of V9281 is quite strong.

\section{CONCLUSION}

This paper aimed at the performance test of metering chip, did key performance testing method research including metering dynamical range, reliability and security, proposed a testing platform for function and performance of metering chips, solved the problem of system deviation caused by whole meter testing traditionally done by electric energy meter manufacturers. The testing platform proposed could load chips from various manufacturers in various packages, and is able to not only evaluate performance of metering chips from different manufacturers but also applied to function and performance test on bulk chips. In additional, the test result of single phase metering chip V9281 proved the platform is practical.

\section{REFERENCES}

[1] Standard of intelligent energy meter series of state grid. Marketing department of state grid, 2013.

[2] J. X. Zhou, Metering function is always the most instinctive quality of energy meter, Metering-china, 2013(3):16-18.

[3] Q. Ch. Fan, F. Zhu, T. Song, M. Yang and Ch. G. Xing, Evaluation of uncertainty about integrated error of electric energy metering, Electrical measurement \& instrumentation, 2009 , 46(525): 76-80.

[4] W. K. Lin, Analysis on abnormal electric quantity involves metering error, Metering-china, 2012(6): 26-27.

[5] L. X. Zhou, Technology research on reliability of intelligent energy meter, Metering-china, 2013(4): 28-30.

[6] Meera Balakrishnan, Security of intelligent meter, Metering-china, 2012(6):32-33.

[7] Data manual of V9281, http://www.vangotech. com.cn. 